\title{
BMJ Open Prevalence of autoimmune thyroid disease in patients with psoriasis: a meta-analysis
}

Xiaochao Zhang, Suhan Zhang, Ruifang Wu, Siying Li, Yuwen Su (D), Peng Zhang

To cite: Zhang X, Zhang S, Wu R, et al. Prevalence of autoimmune thyroid disease in patients with psoriasis: a meta-analysis. BMJ Open 2022;12:e055538. doi:10.1136/ bmjopen-2021-055538

- Prepublication history and additional supplemental material for this paper are available online. To view these files, please visit the journal online (http://dx.doi.org/10.1136/ bmjopen-2021-055538).

$\mathrm{XZ}$ and SZ contributed equally.

Received 16 July 2021 Accepted 11 January 2022

Check for updates

(c) Author(s) (or their employer(s)) 2022. Re-use permitted under CC BY-NC. No commercial re-use. See rights and permissions. Published by BMJ.

Dermatology, The Second Xiangya Hospital of Central South University, Changsha, Hunan, China

Correspondence to Dr Yuwen Su; suyuwen1963@csu.edu.cn and Dr Peng Zhang; zhangpeng@csu.edu.cn

\section{ABSTRACT}

Objective Psoriasis is a chronic inflammatory disease with autoimmune aetiology. A possible link between psoriasis and autoimmune thyroid disease (AITD) has been suggested in some studies with inconsistent findings. This meta-analysis aims to determine the association between psoriasis and AITD.

Design A meta-analysis of observational studies. Data sources PubMed, EMBASE, Scopus and the Cochrane Library were searched up to 1 November 2021. Eligibility criteria for selecting studies We included non-randomised studies, each with over 50 cases in every group, focusing on the rate of comorbidity between psoriasis and AITD.

Data extraction and synthesis Two independent reviewers screened the articles and extracted data. The restricted maximum-likelihood was applied to perform the meta-analysis. OR and 95\% Cls were pooled to compare the prevalence of AITD in psoriasis and control groups. Heterogeneity was assessed with $\mathrm{I}^{2}$ statistic. The Newcastle-Ottawa Scale and Agency for Healthcare Research and Quality were applied for quality assessment. The risk of bias was assessed with Risk Of Bias In Nonrandomised Studies-of Interventions (ROBINS-I).

Results Eleven available studies with data on 253313 patients with psoriasis and 1376533 controls were included. Meta-analysis showed that patients with psoriasis had a higher prevalence of AITD (OR 1.76, $95 \% \mathrm{Cl} 1.35$ to $2.28, \mathrm{Z}=4.25$, $p<0.01$ ), especially loss-of-function disorder of the thyroid gland. Both thyroglobulin antibodies positive rate (OR 1.98, $95 \% \mathrm{Cl} 1.27$ to $3.10, \mathrm{Z}=3.00, \mathrm{p}<0.01$ ) and thyroid peroxidase antibodies positive rate $(\mathrm{OR} 2.15,95 \% \mathrm{Cl} 1.31$ to $3.52, \mathrm{Z}=3.05$, $p<0.01$ ) were also increased in the psoriasis group compared with the control group.

Conclusions Our study indicates that the rate of cooccurring AITD was significantly increased in patients with psoriasis. It suggests that the increased risk of AITD should be concerned in patients with psoriasis.

PROSPERO registration number CRD42020206005.

\section{INTRODUCTION}

Psoriasis is a chronic inflammatory disease with autoimmune aetiology, affecting approximately 125 million people around the world. ${ }^{1}$ The skin lesions of psoriasis occur mainly on the scalp, trunk and exterior surfaces of the limbs, and manifest as erythema, plaques and scales. ${ }^{2}$ Apart from the impaired

\section{Strengths and limitations of this study}

This is the first meta-analysis focusing on the risk of autoimmune thyroid disease (AITD) for psoriasis patients, which included hypothyroidism, hyperthyroidism, subclinical hypothyroidism, subclinical hyperthyroidism, Hashimoto's thyroiditis, Graves' disease, thyroglobulin antibodies positivity and thyroid peroxidase antibodies positivity.

- All studies included were of moderate to high quality and were representative. All studies were published in recent years.

- The heterogeneity in the pooled data cannot be ignored, and was not improved by subgroup analysis. However, meta-regression analysis identified the differences of sample size and scope of research on AITD among these studies as the potential sources of heterogeneity.

appearance and intense pruritus of the skin lesions, various comorbidities have a significant impact on the quality of life in patients with psoriasis. ${ }^{3} 4$ Among the comorbidities, autoimmune thyroid disease (AITD) has been characterised in patients with psoriasis. For patients with psoriasis who also develop complications, their management requires extra attention. ${ }^{5}$ Therefore, understanding the risk of other diseases on psoriasis has important clinical significance.

AITD is an inflammatory disease of the thyroid gland with the presence of thyroid autoantibodies, lymphocytic infiltration of thyroid parenchyma and even thyroid dysfunction. ${ }^{6}$ Graves' disease (GD) and Hashimoto's thyroiditis (HT) are the two main clinical subtypes of AITD. GD is characterised by hyperthyroidism and the presence of thyroid-stimulating hormone receptor antibodies (TRAb) in serum, while HT is characterised by hypothyroidism and the presence of thyroid peroxidase antibodies (TPOAb) or thyroglobulin antibodies (TgAb) in serum. ${ }^{7}$

Psoriasis and AITD share some common pathophysiological features, such as Th1predominant adaptive immune reaction. ${ }^{6}$ 


\begin{tabular}{|c|c|}
\hline Database & Retrieval strategy \\
\hline PubMed & $\begin{array}{l}((((((((((\text { Thyroid(Title/Abstract)) OR (Thyroiditis(Title/Abstract))) OR (Hashimoto Thyroiditis(Title/Abstract))) OR (Graves } \\
\text { Disease(Title/Abstract))) OR (Hyperthyroidism(Title/Abstract))) OR (Hypothyroidism(Title/Abstract))) OR (TgAb(Title/ } \\
\text { Abstract))) OR (TPOAb(Title/Abstract))) OR (TRAb(Title/Abstract))) OR (Endocrine Comorbidities(Title/Abstract))) OR } \\
\text { (Autoimmune diseases(Title/Abstract))) AND ((psoriasis(Title/Abstract)) OR (psoriatic(Title/Abstract))) }\end{array}$ \\
\hline Embase & $\begin{array}{l}\text { (thyroid:ab,ti OR thyroiditis:ab,ti OR 'hashimoto thyroiditis':ab,ti OR 'graves disease':ab,ti OR hyperthyroidism:ab,ti OR } \\
\text { hypothyroidism:ab,ti OR tgab:ab,ti OR tpoab:ab,ti OR trab:ab,ti OR 'endocrine comorbidities':ab,ti OR 'autoimmune } \\
\text { diseases':ab,ti) AND (psoriasis:ab,ti OR psoriatic:ab,ti) }\end{array}$ \\
\hline Scopus & $\begin{array}{l}\text { ((TITLE-ABS-KEY (thyroiditis) OR TITLE-ABS-KEY (hashimoto AND thyroiditis) OR TITLE-ABS-KEY (graves AND } \\
\text { disease) OR TITLE-ABS-KEY (hyperthyroidism) OR TITLE-ABS-KEY (hypothyroidism) OR TITLE-ABS-KEY (tgab) OR } \\
\text { TITLE-ABS-KEY (tpoab) OR TITLE-ABS-KEY (trab))) AND (TITLE-ABS-KEY (psoriasis) OR TITLE-ABS-KEY (psoriatic)) }\end{array}$ \\
\hline Cochrane & $\begin{array}{l}\text { (((Psoriasis) OR psoriatic) OR Pustulosis of Palms) AND (((((((Thyroid) OR Thyroiditis) OR Thyroiditides) OR Hashimoto } \\
\text { Disease) OR Graves Disease) OR Hyperthyroidism) OR Hypothyroidism)). And chose literature published up to } \\
\text { "2021/11/01". Then chose "Trials" }\end{array}$ \\
\hline
\end{tabular}

Hence, the relationship between AITD and psoriasis has been hypothesised and studied. Antonelli et al first reported that the prevalence of AITD in patients with psoriatic arthritis was significantly higher than in the general population. ${ }^{8}$ However, the study reported by Tsai et at pointed that the association between psoriasis and AITD was limited. In recent years, several observational studies regarding the association between psoriasis and AITD were published in succession, but the results of the studies were inconsistent. ${ }^{10-16}$ In addition, Karadag et al reported that the commonly administered acitretin treatment for psoriasis system treatment affects the levels of free T4 (thyroid hormone). ${ }^{17}$ To address this discrepancy, we designed and performed a meta-analysis with the existing evidence to assess the relationship between psoriasis and AITD and provide guidance on the clinical management of psoriasis.

\section{METHODS}

\section{Search strategy}

The literature search was conducted through PubMed, EMBASE, Scopus and the Cochrane Library for relevant studies published before 1 November 2021. Detailed literature-search strategies of the databases are presented in table 1.

\section{Inclusion and exclusion criteria}

The inclusion criteria for the studies included in our analysis were the following: (1) The prevalence of AITD in patients with psoriasis/psoriatic arthritis and nonpsoriasis were studied; (2) The study was a cohort study, case-control study or cross-sectional study; (3) The observed indicators were at least one of the following outcomes: the prevalence of hypothyroidism, hyperthyroidism, HT, GD or the positive rate of TPOAb, TgAb or TRAb; (4) The number of patients with psoriasis and the control group should be over 50. Drug-related studies, animal studies, reviews and conference abstracts were excluded from our analysis.

\section{Data extraction}

The specific process for analysing the studies generated from the search was as follows: record screening and data extraction were performed by two independent authors (XZ and SZ) according to the above retrieval strategy. When disagreements could not be resolved through consensus by the two authors, these were referred to the third author (RW) and resolved through discussion. The following information in the included studies was extracted: the name of the first author, year of publication, country of origin, study design, sample size, the definition of psoriasis and AITD, gender ratio and mean age.

\section{Quality assessment}

The Newcastle-Ottawa Scale (NOS) ${ }^{18}$ was used to assess the quality of the included cohort and case-control studies. The quality of the study was scored on three dimensions: selection, comparability and exposure/outcome. Studies that achieved 0-3, 4-6, 7-9 scores were considered of low, moderate and high quality respectively. Additionally, the tools recommended by the Agency for Healthcare Research and Quality (AHRQ) ${ }^{19}$ were used for the crosssectional studies. Eleven items were included in AHRQ. The study was assigned one point if the answer 'yes', otherwise no points were assigned. Studies that achieved $0-3$, 4-7 and 8-11 points were considered of low, moderate and high quality, respectively. Moreover, the ROBINS-I (Risk Of Bias In Non-randomised Studies-of Interventions) was used to assess the risk of bias. ${ }^{20}$ The assessments were carried by two authors (XZ and SZ), and checked by the third author (RW).

The meta-analysis was performed using Stata V.16.0 software. We used ORs and 95\% CIs to describe the differences between patients with and without psoriasis. Differences were considered statistically significant when $p<0.05$. The prediction interval was used to explore the prevalence of AITD in individuals with psoriasis. The $\mathrm{I}^{2}$ statistic was used to evaluate heterogeneity as follows: $\mathrm{I}^{2} \leq 25 \%$, no heterogeneity; $25 \% \leq \mathrm{I}^{2} \leq 50 \%$, mild heterogeneity; $50 \%<\mathrm{I}^{2} \leq 75 \%$, moderate heterogeneity; $\mathrm{I}^{2}>75 \%$, severe 
heterogeneity. The random effects model was applied throughout the analyses. Publication bias was assessed by funnel plot and Egger's test (publication bias was considered when $\mathrm{p}<0.1$ ). Sensitivity analysis was performed to assess the stability of the meta-analysis by omitting one study in each turn. Univariate meta-regression analysis was used to investigate the sources of heterogeneity. The flow chart was drawn in Adobe Illustrator, and the forest plots, funnel plots, and Egger's test charts were drawn by Stata V.16.0 software.

\section{Patient and public involvement}

No patients or members of the public were involved in this review.

\section{RESULTS}

\section{Search results}

After removing duplicate results, we identified 6380 published studies in the initial search: 6377 studies were included by searching through databases and three studies were harvested by manually searching the references of relevant studies. After screening the titles and abstracts, the remaining 42 studies underwent further full-text screening. Eventually, 11 studies ${ }^{8-1621} 22$ that met the inclusion criteria were included in the final analysis (figure 1).

\section{Study characteristics}

The basic characteristics of the included studies are shown in (tables 2 and 3). A total of 253313 patients with psoriasis and 1376533 control patients were included in the analysis. Two of the studies were cohort studies, eight were case-controlled studies and one was a cross-sectional study.

\section{Quality of studies}

Overall, five studies were of high quality and six of moderate ones (table 3 ). In the studies checked with NOS $(n=10)$, five studies were considered of moderate quality because the control groups were not from the same community. The only cross-sectional study checked

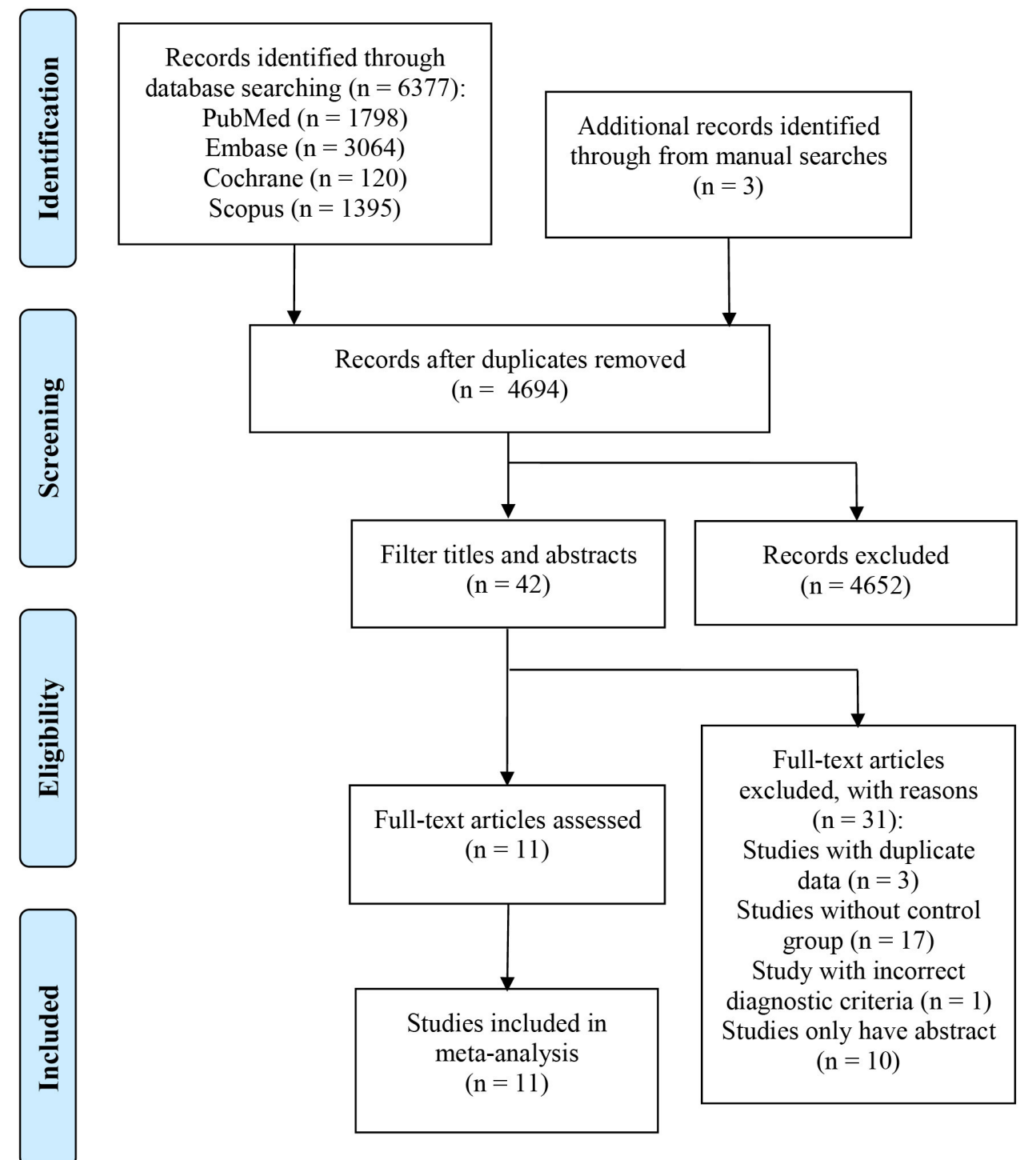

Figure 1 Flow chart for study screening. 
Table 2 Characteristics of the included studies

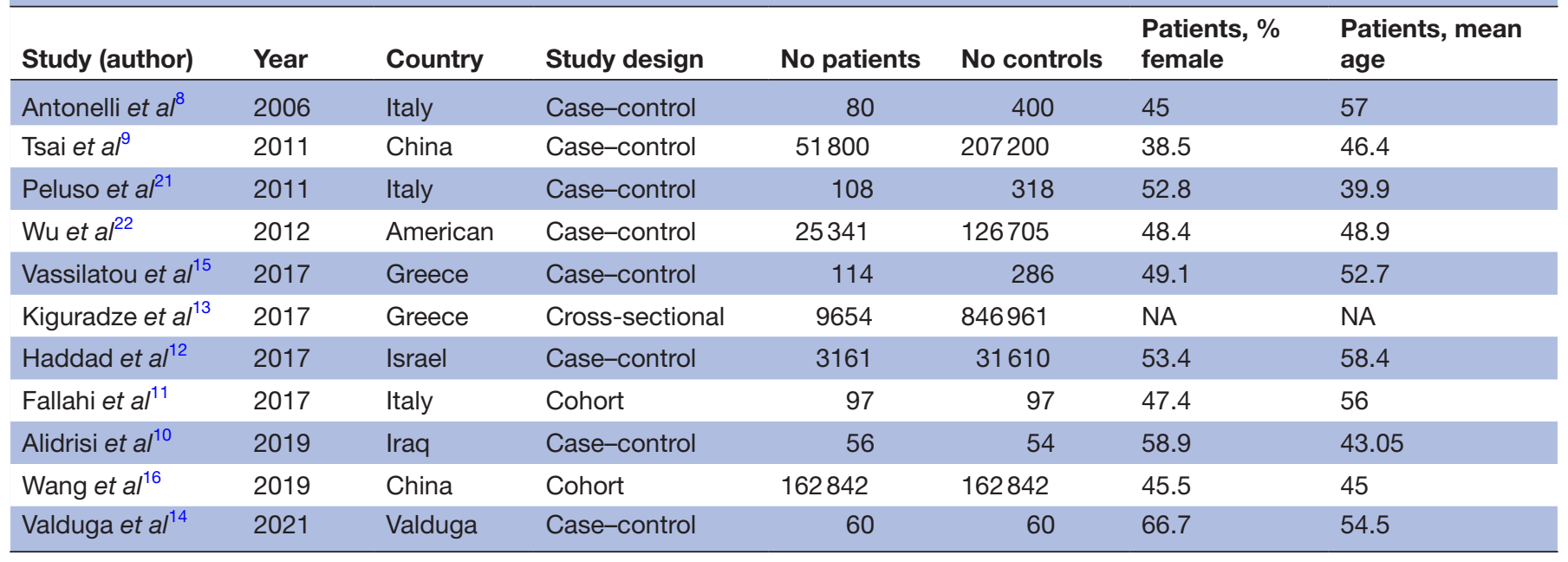

with AHRQ was a moderate quality study as the method for control of confounding was not clear. Based on ROBINS-I, all included studies had moderate risk in overall bias (table 4 ).

\section{Prevalence of AITD in patients with psoriasis}

Eleven studies provided available data on the prevalence of AITD in patients with psoriasis. The meta-analysis showed that patients with psoriasis had a higher prevalence of AITD than the controls (OR 1.76, 95\% CI 1.35 to $2.28, \mathrm{Z}=4.25, \mathrm{p}<0.01)$. The prediction interval ranged from 0.79 to 2.73 , and the heterogeneity was severe $\left(\mathrm{I}^{2}=92.72 \%\right)$.

\section{Heterogeneity analysis}

To investigate potential sources of heterogeneity, we first performed a subgroup analysis by types of study design. The high rate of comorbidity between psoriasis and AITD was also observed in the cross-sectional study strata (OR $2.12,95 \%$ CI 1.55 to 2.89 ), and in the case-control study strata (OR $1.75,95 \%$ CI 1.23 to $2.48, \mathrm{Z}=3.14, \mathrm{p}<0.01$ ) but with heterogeneity remaining severe (figure 2), which indicated that inconsistency of the study designs was not the source of high heterogeneity.

We further conducted a meta-regression analysis to explore the reason for between-study heterogeneity. Seven variables were included in the regression model, covering

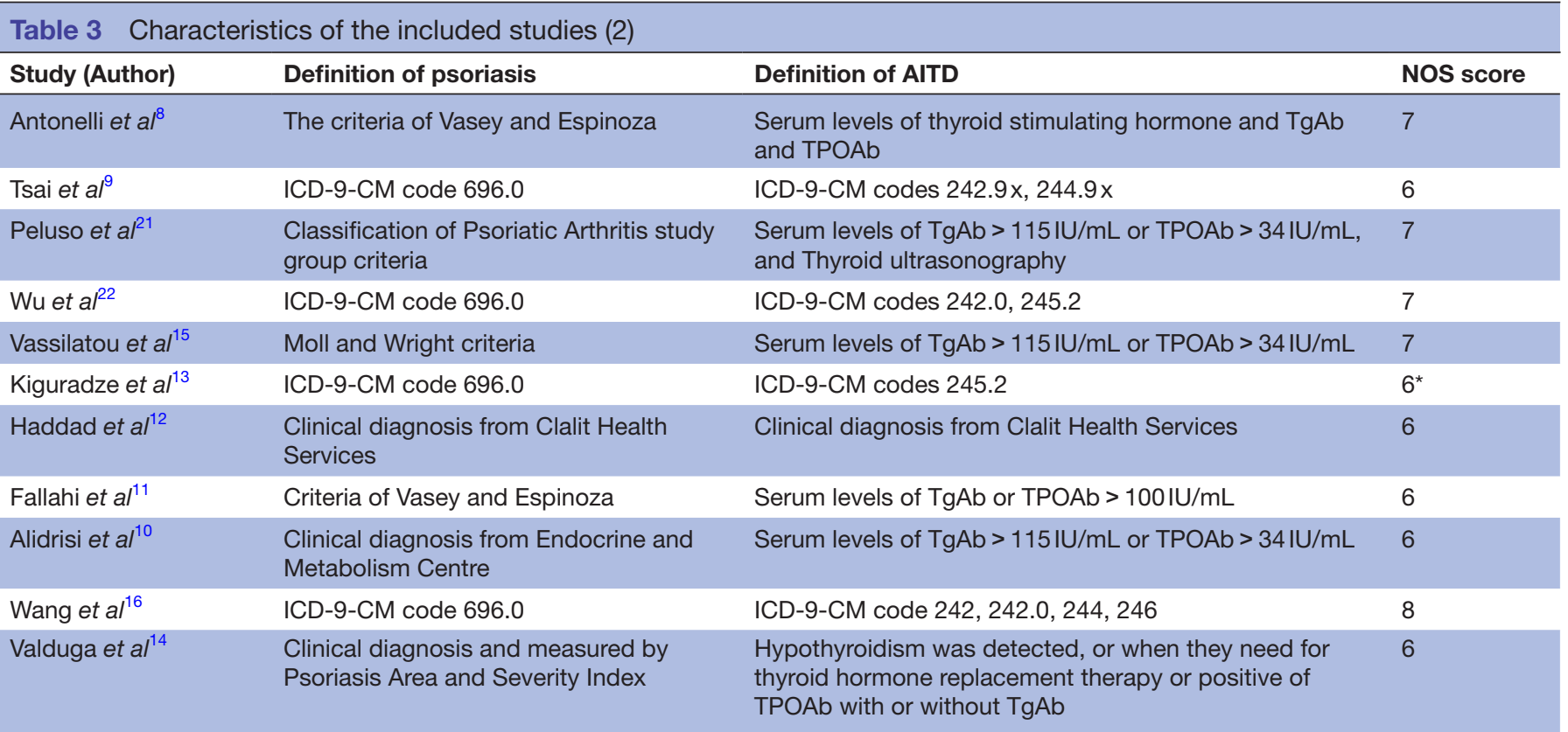

${ }^{*}$ This was a score based on the AHRQ evaluation.

AHRQ, Agency for Healthcare Research and Quality; AITD, autoimmune thyroid disease; ICD-9-CM, International Classification of Diseases, Ninth Revision, Clinical Modification; NOS, Newcastle-Ottawa Scale; TgAb, thyroglobulin antibodies; TPOAb, thyroid peroxidase antibodies. 


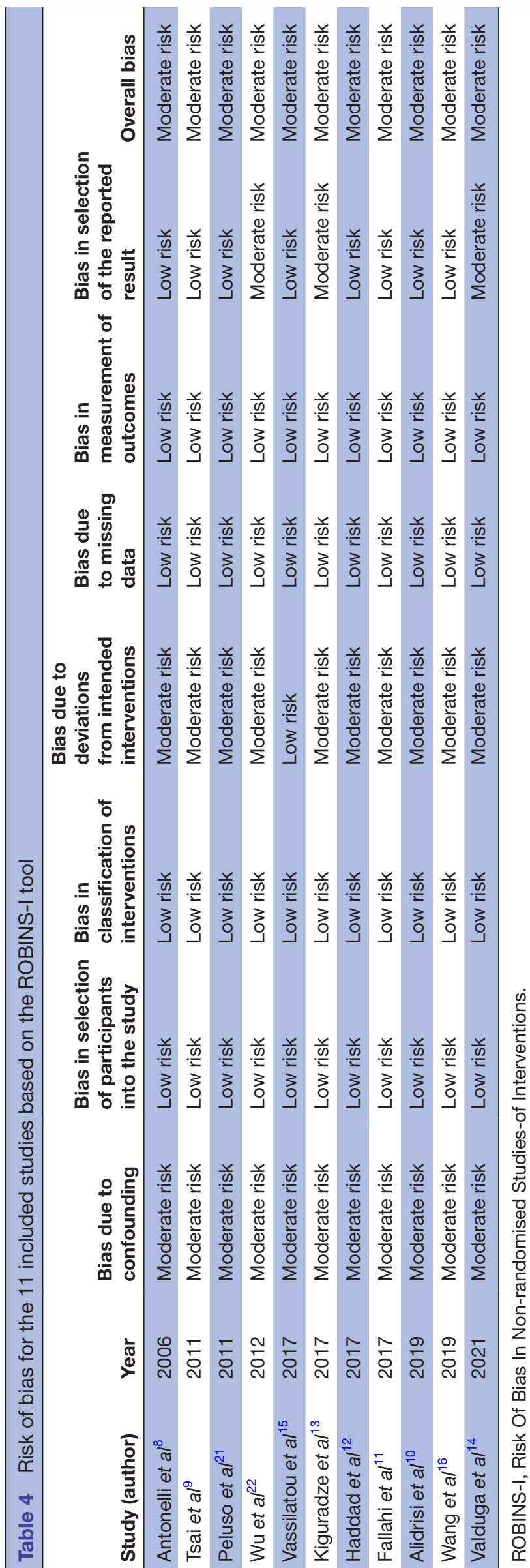

average age, sex ratio, nation (China or other counties), race (Caucasian or non-Caucasian), sample size, clinical types of psoriasis (psoriasis vulgaris or psoriatic arthritis), and scope of research on AITD (all studies were divided into two categories: one focusing on the loss-of-function disorder of the thyroid gland alone and the other focusing on both the loss-of-function disorder and hyperfunction disorder of the thyroid gland). When the statistical significance was set as $\mathrm{p}<0.1$, sample size $(\beta=-0.40, \mathrm{SE}=0.20$, $\mathrm{p}=0.07)$ and scope of research on AITD $(\beta=0.45, \mathrm{SE}=0.15$, $\mathrm{p}=0.02$ ) were the potential sources of high heterogeneity.

We removed three studies ${ }^{81321}$ outside the funnel plot and then conducted the meta-analysis again. The results of the reanalysis also showed that patients with psoriasis had a higher prevalence of AITD than the controls (OR $1.34,95 \%$ CI 1.20 to $1.50, \mathrm{Z}=5.07$, $\mathrm{p}<0.01$ ), but with moderate heterogeneity $\left(\mathrm{I}^{2}=57.30 \%\right)$. The results indicated that these three studies may have contributed to severe heterogeneity in the previous analysis. The source of heterogeneity in the study of Antonelli $e t$ al was that all the patients were patients with psoriatic arthritis, ${ }^{8}$ and the prevalence of AITD in patients with psoriatic arthritis may be higher than that of patients with psoriasis. ${ }^{23}$ The source of heterogeneity in the study by Peluso et al may have been due to the control group being comprised of hospital staff rather than the general population. ${ }^{21}$ The source of heterogeneity in the study of Kiguradze $e t$ al may lie in the fact that it was a cross-sectional study. ${ }^{13}$ These three studies were not excluded because they had little effect on the final results of the analysis.

\section{Sensitivity analysis and publication bias}

Omission of either of the included studies did not significantly change the CI of the combined effect (figure 3A). Therefore, the results of the analysis were considered reliable and stable. The funnel plot for the publication bias is shown in figure 3B. The results of Egger's test showed significant publication bias $(p=0.036$, figure $3 \mathrm{C})$. There was a possibility of exaggerating the association between psoriasis and AITD.

\section{Psoriasis and thyroid function status}

Patients with psoriasis had a higher prevalence of hypothyroidism than the controls (OR 1.21, 95\% CI 1.12 to $1.30, \mathrm{Z}=4.80, \mathrm{p}<0.01)$ and no significant heterogeneity was observed $\left(\mathrm{I}^{2}=0.00 \%\right)$. Patients with psoriasis had a higher prevalence of hyperthyroidism than the controls (OR $1.20,95 \%$ CI 1.12 to $1.30, \mathrm{Z}=4.78, \mathrm{p}<0.01)$ and no significant heterogeneity was observed $\left(\mathrm{I}^{2}=0.00 \%\right.$, figure $\left.4 \mathrm{~A}, \mathrm{~B}\right)$.

Additionally, a higher prevalence of subclinical hypothyroidism and subclinical hyperthyroidism was observed in patients with psoriasis compared with the controls (Subclinical hypothyroidism: OR 2.24, 95\% CI 0.26 to 19.13, $\mathrm{Z}=0.74, \mathrm{p}=0.46$. Subclinical hyperthyroidism: OR $2.66,95 \%$ CI 0.70 to $10.07, \mathrm{Z}=1.44, \mathrm{p}=0.15$ ). However, the difference was not statistically significant (online supplemental figure 1A,B). 


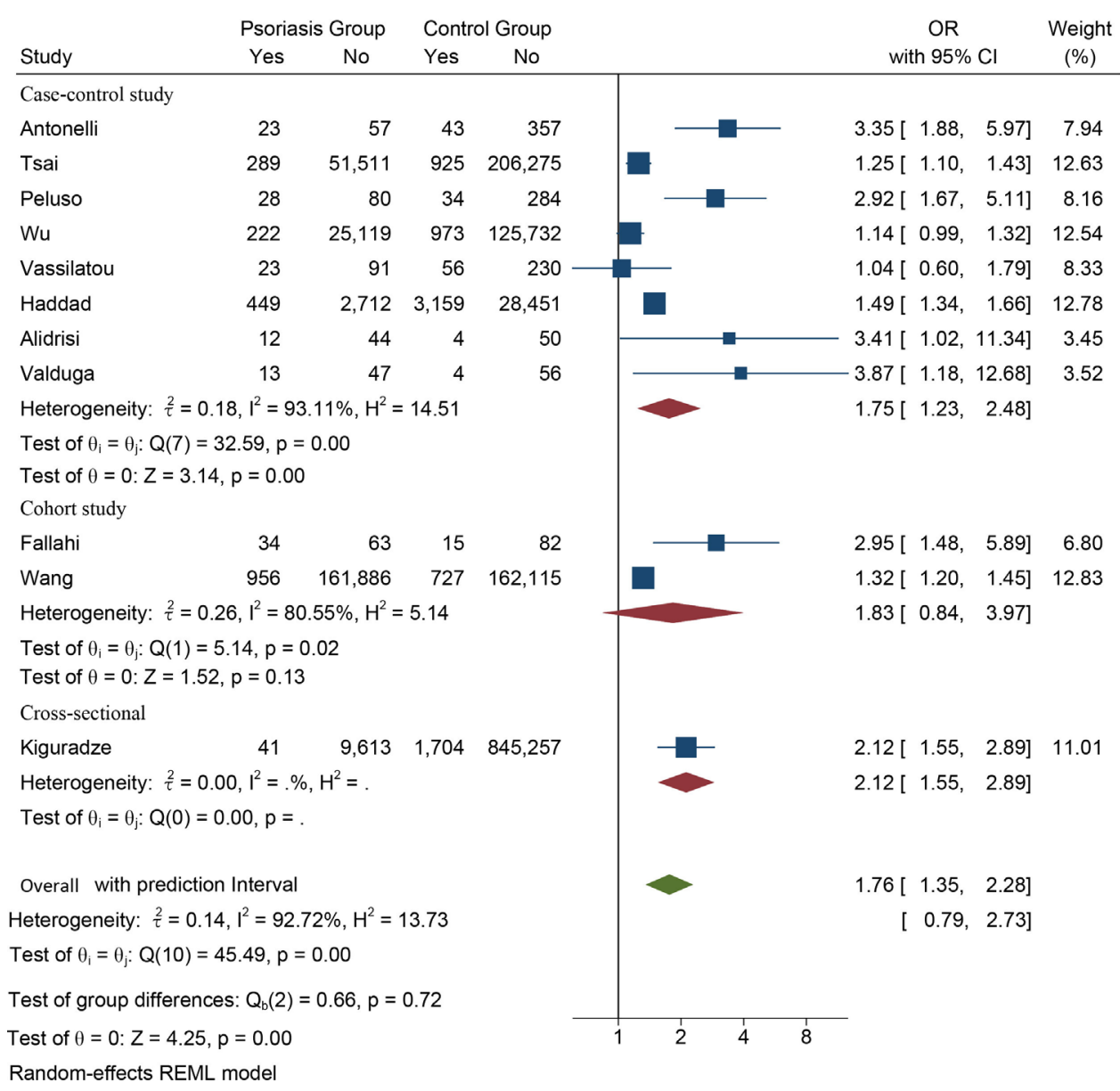

Figure 2 Forest plot of the association between psoriasis and autoimmune thyroid disease.

\section{Psoriasis and specific AITD}

The prevalence of HT in patients with psoriasis and the controls was $0.215 \%$ and $0.199 \%$, respectively. In comparison, the prevalence of GD in patients with psoriasis and the controls was $0.443 \%$ and $0.405 \%$, respectively. The prevalence of HT was significantly higher in patients with psoriasis than the controls (OR 1.88, 95\% CI 1.50 to 2.35 , $\mathrm{Z}=5.47, \mathrm{p}<0.01)$ and the heterogeneity was moderate $\left(\mathrm{I}^{2}=55.98 \%\right)$. The prevalence of GD was also higher in patients with psoriasis than the controls (OR 1.02, 95\% CI 0.65 to $1.60, \mathrm{Z}=0.07, \mathrm{p}=0.94$ ) and the heterogeneity was severe $\left(I^{2}=92.78 \%\right)$. However, the difference was not statistically significant (figures $4 \mathrm{C}$ and $5 \mathrm{~A}$ ).

\section{Psoriasis and thyroid serological antibodies}

No studies provided data on the positive rate of TRAb in patients with psoriasis, so we only included TgAb and $\mathrm{TPOAb}$ in the meta-analysis (figure 5B,C). The positive rate of $\mathrm{TgAb}$ was significantly higher in patients with psoriasis than the controls (OR 1.98, 95\% CI 1.27 to $3.10, \mathrm{Z}=3.00, \mathrm{p}<0.01)$ and the heterogeneity was mild $\left(\mathrm{I}^{2}=41.54 \%\right)$. The positive rate of TPOAb was significantly higher in patients with psoriasis than the controls (OR $2.15,95 \% \mathrm{CI} 1.31$ to $3.52, \mathrm{Z}=3.05, \mathrm{p}<0.01)$ and the heterogeneity was moderate $\left(\mathrm{I}^{2}=56.27 \%\right)$.

\section{DISCUSSION}

To our knowledge, this is the first meta-analysis focusing on the risk of AITD for psoriasis patients. The study by Khan $e t$ al is the first meta-analysis of studies on the association between AITD and the incidence risk of psoriasis. ${ }^{24}$ By summarising all available evidence on the association between psoriasis and AITD, we found that the prevalence of AITD, particularly HT, was higher in patients with psoriasis than the control individuals. Additionally, elevated positive rates of $\mathrm{TgAb}$ and TPOAb were also observed in patients with psoriasis. As psoriasis is a type of discosmetic dermatosis and therefore likely to be of concern, patients with psoriasis are more likely to be active about seeing a doctor regarding their condition than patients with AITD. As such, we recommend that patients with psoriasis receive a thyroid-related examination when they have suspicious AITD-related symptoms. By promoting early diagnosis and treatment of AITD, patients may be able to avoid thyroid dysfunction.

\section{Main findings}

The primary finding of this meta-analysis is that the prevalence of AITD is increased in patients with psoriasis compared with the general population. However, severe heterogeneity was observed. In order to determine whether or not the inconsistency of the study designs 
(A)
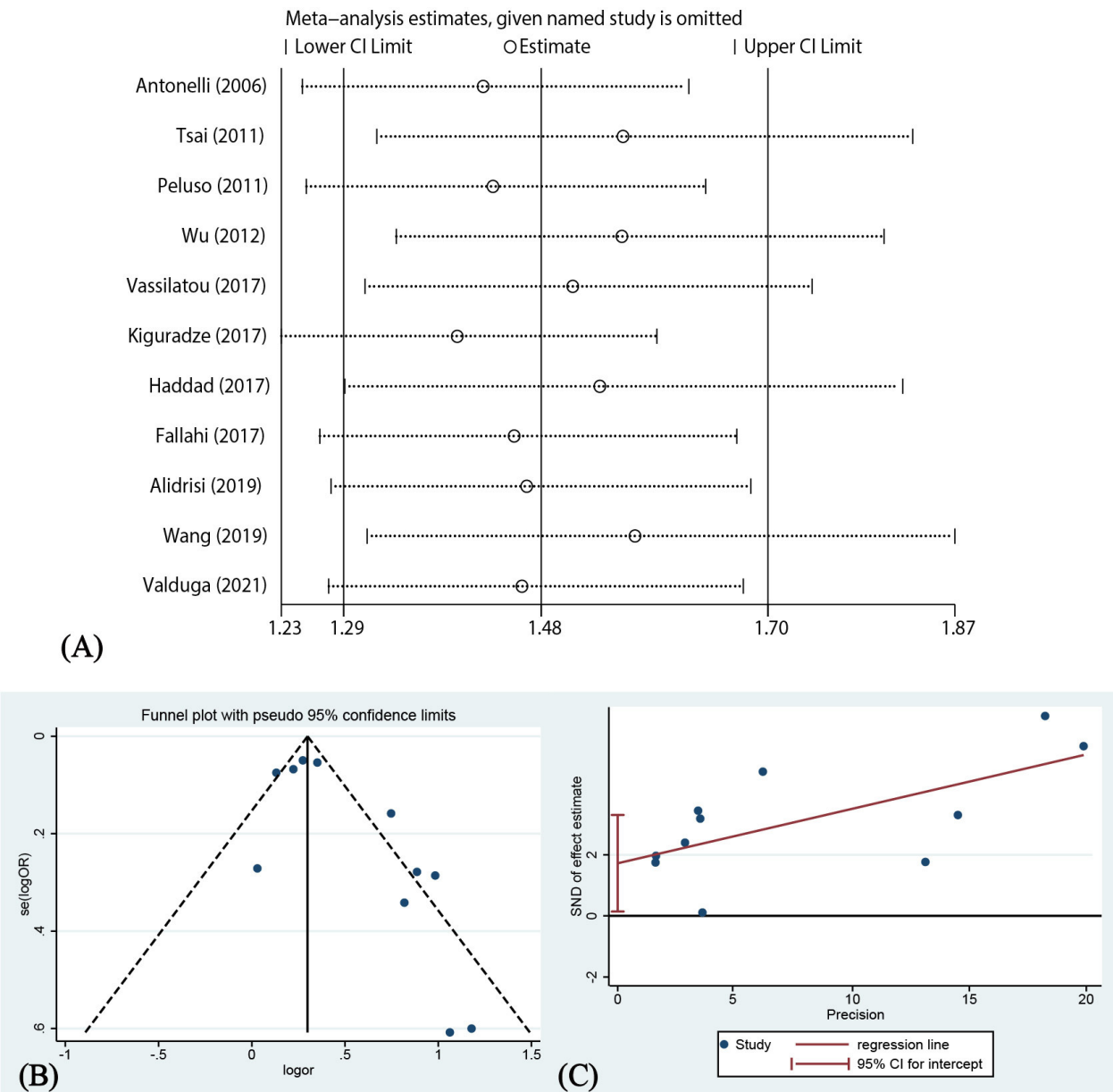

Figure 3 Sensitivity analysis and publication bias. SND, standard normal deviate.

was the primary source of heterogeneity, the subgroup analysis based on different study designs was conducted. However, the heterogeneity was not limited by subgroup analysis; hence the heterogeneity in this meta-analysis was not caused by inconsistency of the study design. Through further meta-regression analysis, we found that the differences in sample size and scope of research on AITD among these studies might explain the high level of between-study heterogeneity. Furthermore, the heterogeneity was improved when we focused on the link of psoriasis with specific clinical characters of AITD, such as hypothyroidism, hyperthyroidism and the positivity rate of autoantibodies.

According to the definition of the study designs, an accurate cause-effect relationship can only be demonstrated in cohort studies. This study included three types of study designs, including cohort, case-controlled and cross-sectional studies. Therefore, the results should be interpreted with caution. Additionally, it has been demonstrated that the prevalence of HT in patients with psoriasis is elevated compared with the controls. HT, a main clinical subtype of AITD, is generally accompanied by hypothyroidism. An elevated frequency of hypothyroidism was also observed in patients with psoriasis. Taken together, the current data indicates that psoriasis may be closely associated with the loss-of-function disorder of the thyroid gland.

\section{Common pathogenesis of psoriasis and AITD}

Both abnormal immunological reactions and underlying genetic risk can contribute to the pathogenesis of psoriasis and AITD. ${ }^{25} 26$ These two diseases share some autoimmune processes and susceptibility genes, which may explain the concurrence of psoriasis and AITD. The predominant Th1 immune reaction has been observed in patients with psoriasis, ${ }^{27}{ }^{28}$ such as Th1 infiltration in involved tissues, and high serum levels of Th1-prototype chemokines and cytokines (TNF- $\alpha$, IFN- $\gamma$ and CXCL10), all of which are present in AITD. ${ }^{29-32}$ Additionally, Th17-mediated immune disorder has also been observed in psoriasis and AITD. ${ }^{33}$ The two diseases share several predisposing genetic alleles or regions. For example, the genetic data from 265 families with two or more autoimmune disorders have shown that the PTPN22-R620W allele has a remarkable association with HT and a mild association with psoriasis. ${ }^{35}$ Additionally, other SNP variations in the PTPN22 gene have been demonstrated to be indicators for evaluating the risk of psoriasis. ${ }^{36}{ }^{37}$ IL12B has been generally recognised as a psoriasis susceptibility gene, ${ }^{38}$ an upstream variation of which affects the phenotype of AITD in men. ${ }^{39}$ 


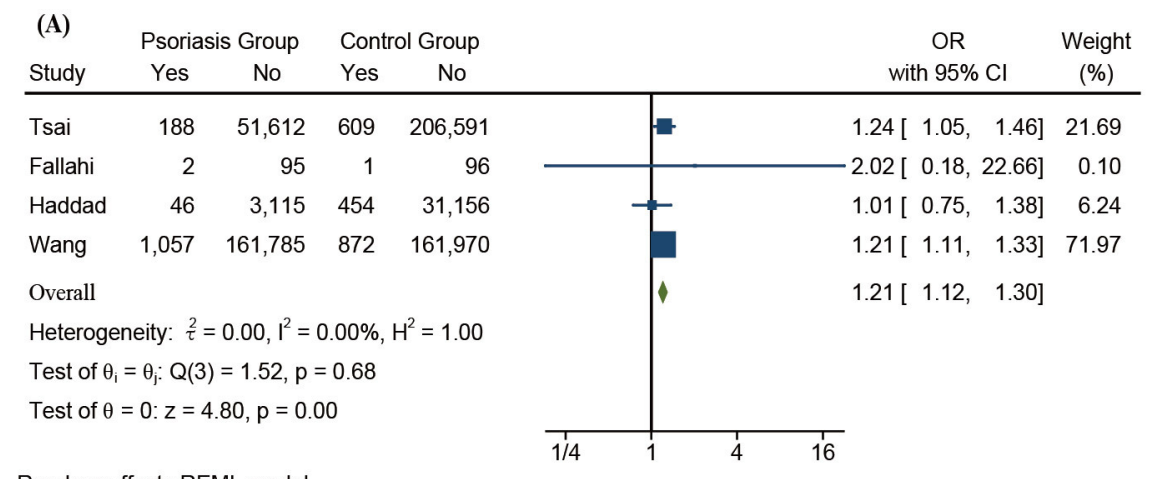

Random-effects REML model

\begin{tabular}{|c|c|c|c|c|c|c|c|}
\hline \multirow{2}{*}{$\begin{array}{l}\text { (B) } \\
\text { Study }\end{array}$} & \multicolumn{2}{|c|}{ Psoriasis Group } & \multicolumn{2}{|c|}{ Control Group } & & \multirow{2}{*}{$\begin{array}{c}\text { OR } \\
\text { with } 95 \% \mathrm{Cl}\end{array}$} & \multirow{2}{*}{$\begin{array}{c}\text { Weight } \\
(\%)\end{array}$} \\
\hline & Yes & No & Yes & No & & & \\
\hline Tsai & 188 & 51,612 & 609 & 206,591 & & $-1.24[1.05,1.46]$ & 21.71 \\
\hline Haddad & 46 & 3,115 & 454 & 31,156 & & $1.01[0.75,1.38]$ & 6.24 \\
\hline Wang & 1,057 & 161,785 & 872 & 161,970 & & $1.21[1.11,1.33]$ & 72.04 \\
\hline \multicolumn{5}{|l|}{ Overall } & & $1.20[1.12,1.30]$ & \\
\hline \multicolumn{8}{|c|}{ Heterogeneity: $\tau^{2}=0.00, \mathrm{I}^{2}=0.00 \%, \mathrm{H}^{2}=1.00$} \\
\hline \multicolumn{8}{|c|}{ Test of $\theta_{\mathrm{i}}=\theta_{\mathrm{j}}: \mathrm{Q}(2)=1.35, \mathrm{p}=0.51$} \\
\hline \multicolumn{8}{|c|}{ Test of $\theta=0: z=4.78, p=0.00$} \\
\hline & & & & 0.75 & 1.00 & 1.46 & \\
\hline
\end{tabular}

Random-effects REML model

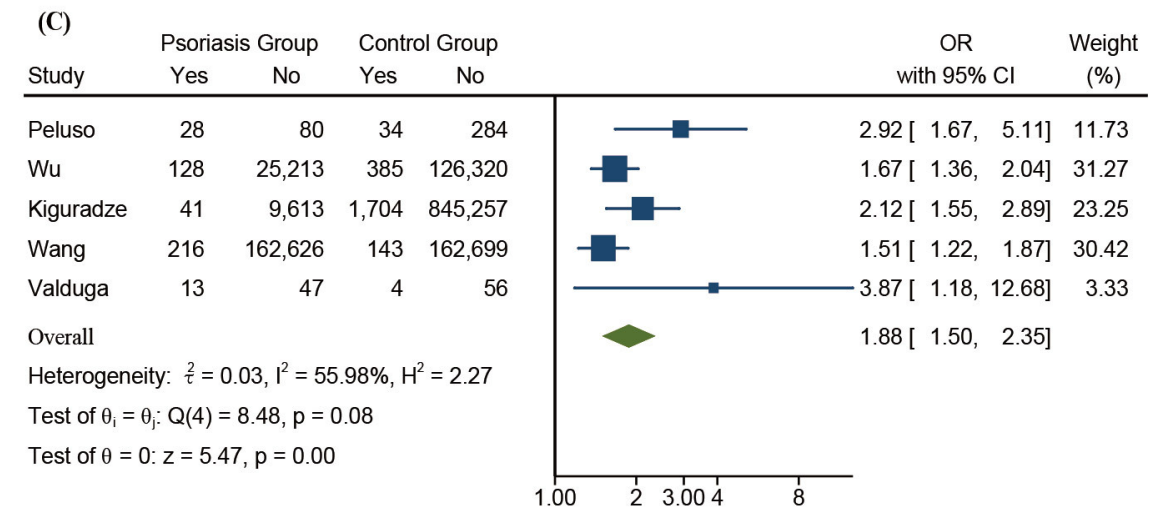

Random-effects REML model

Figure 4 (A) Forest plots of psoriasis and hypothyroidism. (B) Forest plots of psoriasis and hyperthyroidism. (C) Forest plots of psoriasis and HT. HT, Hashimoto's thyroiditis; REML, restricted maximum-likelihood.

\section{Implications for practice}

As information relating to patient medications was not provided by in the original research, drug exposure may be a source of residual confounding in this study and a potential risk factor for concurrence of psoriasis and AITD, apart from the reasons mentioned above. $\beta$-blocker, used to control thyrotoxicosis-related symptoms, has been implicated in induction or exacerbation of psoriasis. ${ }^{40}{ }^{41}$ In addition, it has been reported that the administration of acitretin, a common drug for the treatment of psoriasis, can lead to the reduction of free T4 (thyroid hormone) levels in patients with psoriasis. ${ }^{17}$ Therefore, once the patients develop suspicious symptoms after these treatments, diagnostic investigation and intervention should be considered as early as possible to avoid the exacerbation. On the other hand, the treatment for AITD and psoriasis can be mutually beneficial. For example, propylthiouracil, a drug used to inhibit thyroid hormone synthesis, has been effective in the treatment of psoriasis. ${ }^{42}$ Based on the above findings, it is recommended that the treatment options be adjusted once patients with psoriasis are diagnosed with comorbid AITD.

\section{Limitations}

There are several limitations to this meta-analysis. First, the meta-analysis included studies with different study designs. Given this, we conducted a subgroup analysis of each study design, which also showed that the patients with psoriasis had an increased prevalence of AITD in the cross-sectional study strata and the case-control study strata. Second, there is considerable heterogeneity in this study. Subgroup analysis and meta-regression analysis 


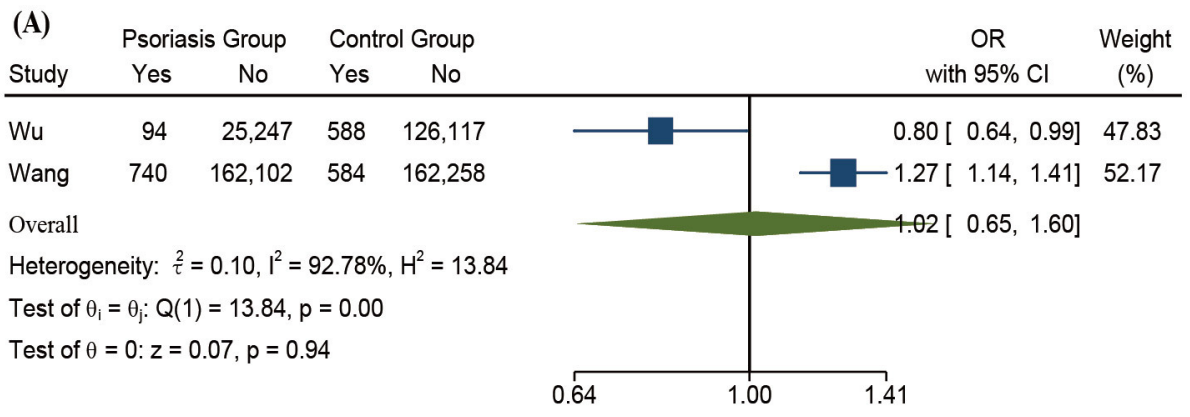

Random-effects REML model

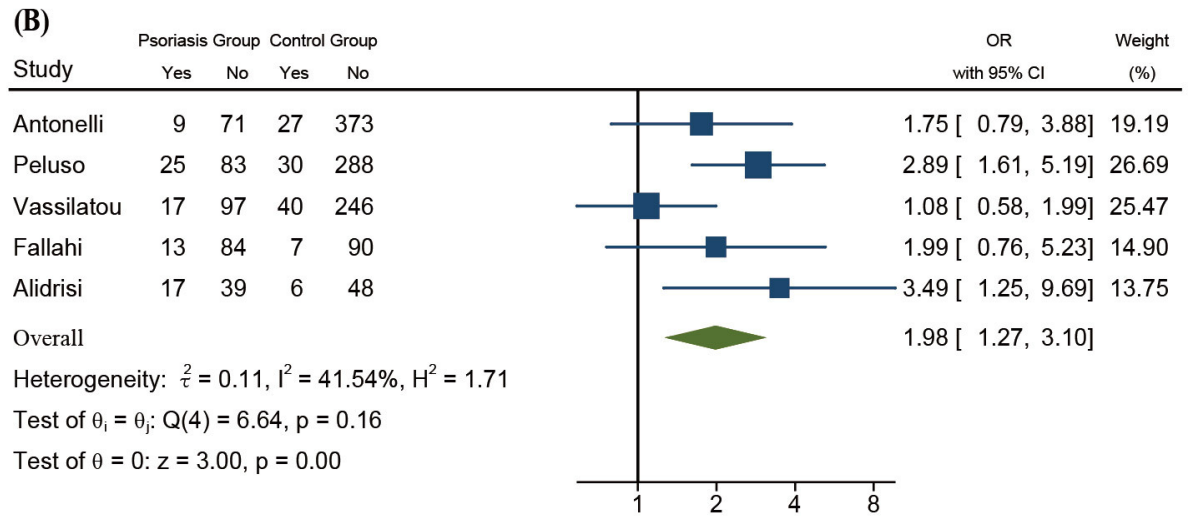

Random-effects REML model

\begin{tabular}{|c|c|c|c|c|c|c|}
\hline \multicolumn{7}{|c|}{ Deriesic C } \\
\hline Study & Yes & No & Yes & No & with $95 \% \mathrm{Cl}$ & $(\%)$ \\
\hline Antonelli & 16 & 64 & 26 & 374 & $3.60[1.83,7.08]$ & 21.60 \\
\hline Peluso & 15 & 93 & 27 & 291 & $1.74[0.89,3.41]$ & 21.70 \\
\hline Vassilatou & 23 & 91 & 54 & 232 & $1.09[0.63,1.87]$ & 25.23 \\
\hline Fallahi & 24 & 73 & 10 & 87 & $2.86[1.28,6.37]$ & 18.57 \\
\hline Alidrisi & 14 & 42 & 5 & 49 & $-3.27[1.09,9.82]$ & 12.91 \\
\hline \multicolumn{5}{|l|}{ Overall } & $2.15[1.31,3.52]$ & \\
\hline \multicolumn{7}{|c|}{ Heterogeneity: $\frac{2}{\tau}=0.17, \mathrm{I}^{2}=56.27 \%, \mathrm{H}^{2}=2.29$} \\
\hline \multicolumn{7}{|c|}{ Test of $\theta_{1}=\theta_{j}: Q(4)=9.40, p=0.05$} \\
\hline \multicolumn{7}{|c|}{ Test of $\theta=0: z=3.05, p=0.00$} \\
\hline & & & & & & \\
\hline
\end{tabular}

Random-effects REML model

Figure 5 (A) Forest plots of psoriasis and GD. (B) Forest plots of psoriasis and TgAb. (C) Forest plots of psoriasis and TPOAb. GD, Graves' disease; REML, restricted maximum-likelihood; TgAb, thyroglobulin antibodies; TPOAb, thyroid peroxidase antibodies.

helped us to identify the potential sources of the heterogeneity. However, there are likely to be other unknown reasons responsible for the heterogeneity. Third, the lack of information on drug application made drug exposure a confounding factor. Therefore, further large-scale and high-quality prospective studies are still required to validate our findings.

\section{CONCLUSIONS}

The present meta-analysis revealed that AITD was more prevalent in patients with psoriasis than in the general population, especially loss-of-function disorder of the thyroid gland. Moreover, patients with psoriasis were found to have elevated positive rates of TPOAb and TgAb compared with the control individuals. Accordingly, we recommend that every dermatologist be conscious of this association and suggest necessary examinations and intervention be considered as soon as possible when patients with psoriasis have suspicious AITD-related symptoms.

Correction notice This article has been corrected since it was published. PZ has been added as co-corresponding author.

Contributors $\mathrm{XZ}$ and $\mathrm{SZ}$ carried out the extraction of reference data, meta-analysis and wrote manuscripts, RW and PZ supported and assisted the manuscripts, YS and SL reviewed and suggested the manuscripts. YS and PZ were responsible for the work and the conduct of the study, controlled the decision to publish. All authors were involved in finalising the manuscript. 
Funding This work was supported by grants from the National Natural Science Foundation of China (No. 82173427 to YS), The 'Xiangya Outstanding Doctor' Bonus and the Fundamental Research Funds for the Central Universities of Central South University (No. 2019zzts905).

Competing interests None declared.

Patient consent for publication Not applicable.

Ethics approval This study does not involve human participants.

Provenance and peer review Not commissioned; externally peer reviewed.

Data availability statement All data relevant to the study are included in the article or uploaded as online supplemental information.

Supplemental material This content has been supplied by the author(s). It has not been vetted by BMJ Publishing Group Limited (BMJ) and may not have been peer-reviewed. Any opinions or recommendations discussed are solely those of the author(s) and are not endorsed by BMJ. BMJ disclaims all liability and responsibility arising from any reliance placed on the content. Where the content includes any translated material, BMJ does not warrant the accuracy and reliability of the translations (including but not limited to local regulations, clinical guidelines, terminology, drug names and drug dosages), and is not responsible for any error and/or omissions arising from translation and adaptation or otherwise.

Open access This is an open access article distributed in accordance with the Creative Commons Attribution Non Commercial (CC BY-NC 4.0) license, which permits others to distribute, remix, adapt, build upon this work non-commercially, and license their derivative works on different terms, provided the original work is properly cited, appropriate credit is given, any changes made indicated, and the use is non-commercial. See: http://creativecommons.org/licenses/by-nc/4.0/.

ORCID iD

Yuwen Su http://orcid.org/0000-0001-9918-1830

\section{REFERENCES}

1 Griffiths CEM, van der Walt JM, Ashcroft DM, et al. The global state of psoriasis disease epidemiology: a workshop report. Br J Dermatol 2017;177:e4-7.

2 Langley RGB, Krueger GG, Griffiths CEM. Psoriasis: epidemiology, clinical features, and quality of life. Ann Rheum Dis 2005;64 Suppl 2:ii18-23.

3 Daudén E, Castañeda S, Suárez C, et al. Clinical practice guideline for an integrated approach to comorbidity in patients with psoriasis. J Eur Acad Dermatol Venereol 2013;27:1387-404.

4 Schaefer CP, Cappelleri JC, Cheng R, et al. Health care resource use, productivity, and costs among patients with moderate to severe plaque psoriasis in the United States. J Am Acad Dermatol 2015;73:585-93.

5 Kaushik SB, Lebwohl MG. Psoriasis: which therapy for which patient: psoriasis comorbidities and preferred systemic agents. J Am Acad Dermatol 2019;80:27-40.

6 Antonelli A, Ferrari SM, Corrado A, et al. Autoimmune thyroid disorders. Autoimmun Rev 2015;14:174-80.

7 Hesarghatta Shyamasunder A, Abraham P. Measuring TSH receptor antibody to influence treatment choices in Graves' disease. Clin Endocrinol 2017;86:652-7.

8 Antonelli A, Delle Sedie A, Fallahi P, et al. High prevalence of thyroid autoimmunity and hypothyroidism in patients with psoriatic arthritis. $J$ Rheumatol 2006;33:2026-8.

9 Tsai T-F, Wang T-S, Hung S-T, et al. Epidemiology and comorbidities of psoriasis patients in a national database in Taiwan. $J$ Dermatol Sci 2011;63:40-6.

10 Alidrisi HA, Al Hamdi K, Mansour AA. Is there any association between psoriasis and Hashimoto's thyroiditis? Cureus 2019;11:e4269.

11 Fallahi P, Ferrari SM, Ruffilli I, et al. Increased incidence of autoimmune thyroid disorders in patients with psoriatic arthritis: a longitudinal follow-up study. Immunol Res 2017;65:681-6.

12 Haddad A, Ashkenazi RI, Bitterman H, et al. Endocrine comorbidities in patients with psoriatic arthritis: a population-based case-controlled study. J Rheumatol 2017;44:786-90.

13 Kiguradze T, Bruins FM, Guido N, et al. Evidence for the association of Hashimoto's thyroiditis with psoriasis: a cross-sectional retrospective study. Int J Dermatol 2017;56:553-6.
14 Valduga JAG, Rebeiko LB, Skare TL. Prevalence of Hashimoto's thyroiditis in psoriasis patients. Rev Assoc Med Bras 2021;67:52-7.

15 Vassilatou E, Papadavid E, Papastamatakis P, et al. No association of psoriasis with autoimmune thyroiditis. J Eur Acad Dermatol Venereol 2017;31:102-6.

16 Wang S-H, Wang J, Lin Y-S, et al. Increased risk for incident thyroid diseases in people with psoriatic disease: a cohort study. J Am Acad Dermatol 2019;80:1006-12

17 Karadag AS, Ozlu E, Kostek O, et al. Effect of low-dose acitretin treatment on pituitary hormones in psoriasis vulgaris: a retrospective study. Indian J Dermatol Venereol Leprol 2019;85:300-4.

18 Wells G, Shea B, O'connell D. The Newcastle-Ottawa scale (NOS) for assessing the quality of nonrandomised studies in Newcastleanalyses, 2019. Available: http://www.ohri.ca/programs/clinical_ epidemiology/oxford.asp

19 Rostom A, Dubé C, Cranney A. AHRQ evidence reports, 2004. Available: https://www.ncbi.nlm.nih.gov/books/NBK35156/

20 Sterne JA, Hernán MA, Reeves BC, et al. ROBINS-I: a tool for assessing risk of bias in non-randomised studies of interventions. BMJ 2016;355:i4919.

21 Peluso R, Lupoli GA, Del Puente A, et al. Prevalence of thyroid autoimmunity in patients with spondyloarthropathies. J Rheumatol 2011;38:1371-7

$22 \mathrm{Wu} J \mathrm{JJ}$, Nguyen TU, Poon K-YT, et al. The association of psoriasis with autoimmune diseases. J Am Acad Dermatol 2012;67:924-30.

23 Vastarella M, Megna M, Lupoli GA, et al. Is there any association between psoriasis, psoriatic arthritis and thyroid autoimmunity? Australas J Dermatol 2021;62:e207-e211.

24 Khan SR, Bano A, Wakkee M, et al. The association of autoimmune thyroid disease (AITD) with psoriatic disease: a prospective cohort study, systematic review and meta-analysis. Eur J Endocrinol 2017;177:347-59.

25 Perera GK, Di Meglio P, Nestle FO. Psoriasis. Annu Rev Pathol 2012;7:385-422

26 Simmonds MJ. Gwas in autoimmune thyroid disease: redefining our understanding of pathogenesis. Nat Rev Endocrinol 2013;9:277-87.

27 Cai Y, Fleming C, Yan J. New insights of T cells in the pathogenesis of psoriasis. Cell Mol Immunol 2012;9:302-9.

28 Lowes MA, Suárez-Fariñas M, Krueger JG. Immunology of psoriasis. Annu Rev Immunol 2014;32:227-55.

29 Antonelli A, Ferrari SM, Giuggioli D, et al. Chemokine (C-X-C motif) ligand (CXCL) 10 in autoimmune diseases. Autoimmun Rev 2014;13:272-80.

30 Ruffilli I, Ragusa F, Benvenga S, et al. Psoriasis, psoriatic arthritis, and thyroid autoimmunity. Front Endocrinol 2017;8:139.

31 Nanba T, Watanabe M, Inoue N, et al. Increases of the Th1/Th2 cell ratio in severe Hashimoto's disease and in the proportion of Th17 cells in intractable Graves' disease. Thyroid 2009;19:495-501.

32 Peng S, Li C, Wang X, et al. Increased Toll-like receptors activity and TLR ligands in patients with autoimmune thyroid diseases. Front Immunol 2016;7:578

33 Hänsel A, Günther C, Ingwersen J, et al. Human slan (6-sulfo lacNAc) dendritic cells are inflammatory dermal dendritic cells in psoriasis and drive strong TH17/TH1 T-cell responses. J Allergy Clin Immunol 2011;127:787-94.

34 Vitales-Noyola M, Ramos-Levi AM, Martínez-Hernández R, et al. Pathogenic Th17 and Th22 cells are increased in patients with autoimmune thyroid disorders. Endocrine 2017:57:409-17.

35 Criswell LA, Pfeiffer KA, Lum RF, et al. Analysis of families in the multiple autoimmune disease genetics Consortium (MADGC) collection: the PTPN22 620W allele associates with multiple autoimmune phenotypes. Am J Hum Genet 2005;76:561-71.

$36 \mathrm{Li} \mathrm{Y,} \mathrm{Liao} \mathrm{W,} \mathrm{Chang} \mathrm{M,} \mathrm{et} \mathrm{al.} \mathrm{Further} \mathrm{genetic} \mathrm{evidence} \mathrm{for} \mathrm{three}$ psoriasis-risk genes: ADAM33, CDKAL1, and PTPN22. J Invest Dermatol 2009;129:629-34.

37 Wang $\mathrm{H}$, Wang Z, Rani PL, et al. Identification of PTPN22, ST6GAL1 and Jazf1 as psoriasis risk genes demonstrates shared pathogenesis between psoriasis and diabetes. Exp Dermatol 2017;26:1112-7.

38 Cargill M, Schrodi SJ, Chang M, et al. A large-scale genetic association study confirms IL12B and leads to the identification of IL23R as psoriasis-risk genes. Am J Hum Genet 2007;80:273-90.

39 Walsh JP, Berry J, Liu S, et al. The clinical presentation of autoimmune thyroid disease in men is associated with IL12B genotype. Clin Endocrinol 2011;74:508-12.

40 Balak DM, Hajdarbegovic E. Drug-Induced psoriasis: clinical perspectives. Psoriasis 2017;7:87-94.

41 McDermott MT. Hyperthyroidism. Ann Intern Med 2020;172:ITC49.

42 Gnanaraj P, Malligarjunan H, Dayalan H, et al. Therapeutic efficacy and safety of propylthiouracil in psoriasis: an open-label study. Indian J Dermatol Venereol Leprol 2011;77:673-6. 